\title{
On the Asymptotic Behavior of Second Order Quasilinear Difference Equations
}

\author{
Vadivel Sadhasivam1, Pon Sundar ${ }^{2}$, Annamalai Santhi' \\ ${ }^{1} \mathrm{PG}$ and Research Department of Mathematics, Thiruvalluvar Government Arts College, Rasipuram, Namakkal, \\ India \\ ${ }^{2}$ Om Muruga College of Arts and Science, Salem, India \\ Email: ovsadha@gmail.com,mydt972009@gmail.com,parimalam_sss@yahoo.co.in
}

Received 15 June 2016; accepted 26 August 2016; published 29 August 2016

Copyright (C) 2016 by authors and Scientific Research Publishing Inc.

This work is licensed under the Creative Commons Attribution International License (CC BY).

http://creativecommons.org/licenses/by/4.0/

(c) (i) Open Access

\section{Abstract}

In this paper, we investigate the asymptotic behavior of the following quasilinear difference equations

$$
\Delta\left(|\Delta y(n)|^{\alpha-1} \Delta y(n)\right)=p(n)|y(n)|^{\beta-1} y(n),
$$

where $n \in N_{0}=\left\{n_{0}, n_{0}+1, n_{0}+2, \cdots\right\}, n_{0} \in N$. We classified the solutions into six types by means of their asymptotic behavior. We establish the necessary and/or sufficient conditions for such equations to possess a solution of each of these six types.

\section{Keywords}

Asymptotic Behavior, Positive Solutions, Homogeneous, Quasilinear Difference Equations

\section{Introduction}

Recently, the asymptotic properties of the solutions of second order differential equations [1] [2] difference equations of the type (E) and/or related equations have been investigated by many authors, for example see, [3]-[19] and the references cited there in. Following this trend, we investigate the existence of these six types of solutions of the Equation (E) showing the necessary and/or sufficient conditions can be obtained for the existence of those solutions. For the general backward on difference equations, the reader is referred to the monographs [20]-[24].

In 1996, PJY Wang and R.P. Agarwal [25] considered the quasilinear equation

$$
\Delta\left(a_{n-1}\left(\Delta y_{n-1}\right)^{\sigma}\right)+q_{n} f\left(y_{n}\right)=0
$$


and obtained oscillation criteria for the Equation (1).

In 1996, E. Thandapani, M.M.S. Manuel and R.P. Agarwal [26] have studied the quasi-linear difference equation

$$
\Delta\left(a_{n-1}\left|\Delta x_{n-1}\right|^{\alpha-1} \Delta x_{n-1}\right)+q_{n} f\left(x_{n}\right)=0 .
$$

In 2000, Pon Sundaram and E. Thandapani [27] considered the following quasi-linear functional difference equation

$$
\Delta\left(|\Delta y(n)|^{\alpha-1} \Delta y(n)\right)+f(n, y(\sigma(n)))=0
$$

and they have established necessary and sufficient conditions for the solutions of Equation (3) to have various types of nonoscillatory solutions. Further they have established some new oscillation conditions for the oscillation of solutions of Equation (3).

In 1997, E. Thandapani and R. Arul [28] studied, the following quasi-linear equation

$$
\Delta\left(p_{n} \phi\left(y_{n}\right)\right)+f\left(n, y_{n+1}\right)=0 .
$$

They established necessary and sufficient conditions for the solutions of (4) to have various type of nonoscillatory solutions.

In 2004, E. Thandapani et al. [29] studied the equation

$$
\Delta\left(a_{n} \Delta\left(y_{n}-p y_{n-k}^{\alpha}\right)\right)+q_{n} f\left(y_{n-\ell+1}\right)=0, \quad n \geq n_{0} \geq 0,
$$

and established conditions for the existence of non-oscillatory solutions.

S.S. Cheng and W.T. Patula [30] studied the difference equation

$$
\Delta\left(\Delta y_{k-1}\right)^{p-1}+s_{k} y_{k}^{p-1}=0
$$

where $p>1$ and proved an existence theorem for Equation (6).

In 2002, M. Mizukanmi et al. [1] discussed the asymptotic behavior of the following equation

$$
\left(\left|y^{\prime}\right|^{\alpha-1} y^{\prime}\right)=p(t)|y|^{\beta-1} y \text {. }
$$

Discrete models are more suitable for understanding the problems in Economics, genetics, population dynamics etc. In the qualitative theory of difference equations asymptotic behavior of solutions plays a vital role. Motivated by this, we consider the discrete analogue of (7) of the form

$$
\Delta\left(|\Delta y(n)|^{\alpha-1} \Delta y(n)\right)=p(n)|y(n)|^{\beta-1} y(n)
$$

where $n \in N_{0}=\left\{n_{0}, n_{0}+1, n_{0}+2, \cdots\right\}, n_{0} \in N$ and $\Delta$ is the forward difference operator defined by

$$
\Delta y(n)=y(n+1)-y(n) .
$$

We assume the following conditions on Equation (8)

1) $\alpha$ and $\beta$ are positive constants

2) $\{p(n)\}$ is a real sequence such that $p(n)>0$ for all $n \geq n_{0}>0$.

For simplicity, we often employ the notation

$$
x^{\gamma^{*}}=|x|^{\gamma-1} x=|x|^{\gamma} \operatorname{sgn} x, \quad x \in R, \quad \gamma>0
$$

interms of which Equation (8) can be expressed in

$$
\Delta(\Delta y(n))^{\alpha^{*}}=p(n)(y(n))^{\beta^{*}} .
$$

By a solution of Equation (8), we mean a real sequence $y(n): N_{0} \rightarrow R$, together with $|\Delta y(n)|^{\alpha-1} y(n)$ exists and satisfies Equation (8) for all $n \geq n_{0} \in N_{0}$.

We here call Equation (8) super-homogeneous or sub-homogeneous according as $\alpha<\beta$ or $\alpha>\beta$ If $\alpha=\beta$ Equation (8) is often called half-linear. Our attention is mainly paid to the super-homogeneous and sub-homo- 
geneous cases, and the half-linear is almost excluded from our consideration.

\section{The Classification of All Solutions of Equation (8)}

To classify all solutions of Equation (8), we need the following lemma.

Lemma 1. Let $y(n)$ be a local solutions of Equation (8) near $n=N \geq n_{0}$ and $[N, w), w \leq \infty$, be its right maximal interval of existence. Then we have either $y(n) \geq 0$ near $w$ or $y(n) \leq 0$ near $w$. That is $y(n)$ does not charge strictly its sign infinitely many times as $n \uparrow w$.

The classification of all (local) solutions of Equation (8) are given on the basis of Lemma 1. Since the proof is easy, we leave it to the reader.

Proposition 1. Each local solution $y(n) \neq \equiv$ of Equation (8) falls into exactly one of the following six types.

1) Singular solution of the first kind: type $\left(S_{1}\right)$ there exist a $n_{1} \geq n_{0}$ such that

$$
y(n) \neq 0 \text { for } n \leq n_{1} \text {, and } y(n) \equiv 0 \text { for } n \geq n_{1} .
$$

2) Decaying solution: type (D), $y(n)$ can be continued to $\infty$, and satisfies $y(n) \Delta y(n)<0$ for all large $n$, and

$$
\lim _{n \rightarrow \infty} y(n)=0 .
$$

3) Asymptotically constant solution: type (AC) $y(n)$ can be continued to $\infty$, and satisfies $y(n) \Delta y(n)<0$ for all large $n$ and

$$
\lim _{n \rightarrow \infty} y(n) \in R-\{0\} .
$$

4) Asymptotically linear solution: type (AL) $y(n)$ can be continued to $\infty$ and satisfies $y(n) \Delta y(n)>0$ for all large $n$ and

$$
\lim _{n \rightarrow \infty} \frac{y(n)}{n} \in R-\{0\} .
$$

5) Asymptotically super-linear solution: type (AS) $y(n)$ can be continued to $\infty$ and satisfies $y(n) \Delta y(n)>0$ for all large $n$ and

$$
\lim _{n \rightarrow \infty} \frac{y(n)}{n}= \pm \infty \text {. }
$$

6) Singular solution of second kind: type $\left(S_{2}\right) \quad y(n)$ has the finite escape time; that is, there exists a $n_{1}>n_{0}$ such that

$$
\lim _{n \rightarrow \infty} y(n)= \pm \infty
$$

\section{Main Results for the Super-Homogeneous Equations}

Before we list our main results for the case $\alpha<\beta$. Throughout this section we assume that $\alpha<\beta$.

Theorem 2. Equation (8) has no solution of type $\left(S_{1}\right)$.

Theorem 3. Equation (8) has a solution of type (D) if and only if

$$
\sum^{\infty}\left(\sum_{n}^{\infty} p(s)\right)^{1 / \alpha}=\infty
$$

Theorem 4. Equation (8) has a solution of type (AC) if and only if

$$
\sum^{\infty}\left(\sum_{n}^{\infty} p(s)\right)^{1 / \alpha}<\infty .
$$

Theorem 5. Equation (8) has a solution of type $(A L)$ if and only if

$$
\sum^{\infty} n^{\beta} p(n)<\infty .
$$


Theorem 6. Equation (8) has a solution of type (AS) if (11) holds.

Theorem 7. Equation (8) does not have solutions of type (AS) if there are constants $\rho>0$ and $\sigma \in(0,1)$ satisfying

$$
\liminf _{n \rightarrow \infty} n^{\rho} \sum_{n}^{\infty} s^{\beta \sigma+\sigma-\rho-1}(p(s))^{\alpha}>0
$$

and

$$
\left.\begin{array}{l}
\beta \sigma+\sigma-\rho-1 \geq 0 \\
1-\sigma-\alpha \sigma-\alpha \rho \geq 0
\end{array}\right\}
$$

Remark 1. The set of all pairs $(\rho, \tau) \in(0, \infty) \times(0,1)$ satisfying inequalities (13) is not empty. In fact, the pair $(\rho, \sigma)=\left(\frac{\beta-\alpha}{\alpha \beta+2 \alpha+1}, \frac{\alpha+1}{\alpha \beta+2 \alpha+1}\right)$ belongs to it.

Theorem 8. Equation (8) has a solutions of type $\left(S_{2}\right)$.

Remark 2. Theorem 7 has the same conclusion that these are not solutions of type (AS). However, Theorem 7 is still valid for the case that $p$ is nonnegative. For example, it is formed by this extended version of Theorem 7 that the equation

$$
\Delta\left(|\Delta y(n)|^{\alpha-1} \Delta y(n)\right)=(1+t)|y(n)|^{\beta-1} y(n), \quad n \geq 1
$$

does not have solutions of type (AS).

Example 1 Let $\alpha<\beta$, consider the Equation (8) with $p(n)=n^{\sigma}$

$$
\Delta\left(|\Delta y(n)|^{\alpha-1} \Delta y(n)\right)=n^{\alpha}|y(n)|^{\beta-1} y(n), n \geq 1 \text { and } \sigma \in R .
$$

For this equation, we have the following results:

1) Equation (14) has a solution of type (D) if and only if $\sigma \geq-\alpha-1$ (Theorem 3).

2) Equation (14) has a solution of type (AC) if and only if $\sigma<-\alpha-1$ (Theorem 4).

3 ) Equation (14) has a solution of type (AL) if and only if $\sigma<-\beta-1$ (Theorem 5).

4) Equation (14) has a solution of type (AS) if and only if $\sigma<-\beta-1$ (Theorem 6).

\section{Main Results for the Sub-Homogeneous Equation}

Below we list our main results for the case $\alpha>\beta$. Throughout this section we assume that $\alpha>\beta$.

Theorem 9. Equation (8) has a solutions of type $\left(S_{1}\right)$.

Theorem 10. Equation (8) has a solution of type (D) if

$$
\sum^{\infty}\left(\sum_{n}^{\infty} p(s)\right)^{1 / \alpha}<\infty .
$$

Theorem 11. Equation (8) does not have solutions of type $(D)$ if

$$
\liminf _{n \rightarrow \infty} n^{1+\alpha} p(n)>0 .
$$

Theorem 12. Equation (8) does not have solutions of type $(D)$ if there are constants $\rho>0$ and $\sigma \in(0,1)$ satisfying

$$
\liminf _{n \rightarrow \infty} n^{\rho \alpha} \sum_{n}^{\infty}(s)^{\sigma+\alpha \sigma-\alpha \rho-1}|p(s)|>0
$$

and

$$
\left\{\begin{array}{l}
\beta \sigma+\sigma+\rho-1 \leq 0 \\
1-\sigma-\sigma \alpha+\alpha \rho \leq 0 .
\end{array}\right.
$$

Remark 3. The set of all pairs $(\rho, \sigma) \in(0, \infty) \times(0,1)$ satisfying inequalities (18) is not empty. In fact, the 
pair

$$
(\rho, \sigma)=\left(\frac{\alpha-\beta}{\alpha \beta+2 \alpha+1}, \frac{\alpha+1}{\alpha \beta+2 \alpha+1}\right)
$$

belongs to it.

Theorem 13. Equation (8) has a solution of type (AC) if and only if (15) holds.

Theorem 14. Equation (8) has a solution of type $(A L)$ if and only if

$$
\sum^{\infty} n^{\beta} p(n)<\infty
$$

Theorem 15. Equation (8) has a solution of type (AS) if and only if

$$
\sum^{\infty} n^{\beta} p(n)=\infty .
$$

Theorem 16. Equation (8) has no solutions of type $\left(S_{2}\right)$.

Example 2. Let $\alpha>\beta$ and consider the Equation (14) again.

We have the following results:

1) Equation (14) has a solution of type (D) if and only if $\sigma<-\alpha-1$ (Theorem 10 and 11).

2) Equation (14) has a solution of type (AC) if and only if $\sigma<-\alpha-1$ (Theorem 14).

3) Equation (14) has a solution of type ( $A L$ ) if and only if $\sigma<-\beta-1$ (Theorem 15).

4) Equation (14) has a solution of type (AS) if and only if $\sigma \geq-\beta-1$ (Theorem 16).

\section{Auxillary Lemma}

In this section, we collect axillary lemmas, which are mainly concerned with local solution of Equation (8). A comparison lemma of the following type is useful, and will be used in many places.

Lemma 2. Suppose that $\left\{p_{p}(n)\right\},\left\{p_{q}(n)\right\}$ are such that $0<p_{1}(n)<p_{2}(n)$ for $a \leq n \leq b$. Let $y_{i}(n)$, $i=1,2$ and $a \leq n \leq b$ be solutions of the equations

$$
\Delta\left(\left|\Delta y_{i}(n)\right|^{\alpha-1} \Delta y_{i}(n)\right)=p_{i}(n)\left|y_{i}(n)\right|^{\beta-1} y_{i}(n), i=1,2
$$

respectively. If $y_{1}(a) \leq y_{2}(a)$ and $\Delta y_{1}(a) \leq \Delta y_{2}(a)$, then $y_{1}(n)<y_{2}(n)$ and $\Delta y_{1}(n)<\Delta y_{2}(n)$ for $a<n$ $\leq b$.

Proof. We have

$$
\begin{aligned}
& \left(\Delta y_{i}(n)\right)^{\alpha_{*}}=\left(\Delta y_{i}(a)\right)^{\alpha_{*}}+\sum_{a}^{n-1} p_{i}(s)\left(y_{i}(s)\right)^{\beta_{*}}, a \leq n \leq b, i=1,2 \\
& y_{i}(n)=y_{i}(a)+\sum_{a}^{n-1}\left(\left(\Delta y_{i}(a)\right)^{\alpha_{*}}+\sum_{a}^{s-1} p_{i}(r)\left(y_{i}(r)\right)^{\beta_{*}}\right)^{1 / \alpha^{*}}, a \leq n \leq b, i=1,2 .
\end{aligned}
$$

By the hypotheses we have $y_{1}(n)<y_{2}(n)$ in some right neighborhood of $a$. If $y_{1}(n) \geq y_{2}(n)$ for some point in $a<n \leq b$, we can find a $c$ such that $a<c \leq b$ satisfying $y_{1}(n)<y_{2}(n)$ for $a<n<c$ and $y_{1}(c)=y_{2}(c)$. But, this yields a contradiction, because

$$
\begin{aligned}
0= & y_{1}(c)-y_{2}(c) \\
= & y_{1}(a)-y_{2}(a)+\sum_{a}^{c-1}\left[\left(\left(\Delta y_{1}(a)\right)^{\alpha_{*}}+\sum_{a}^{n-1} p_{1}(s)\left(y_{1}(s)\right)^{\beta_{*}}\right)^{1 / \alpha^{*}}\right. \\
& \left.-\left(\left(\Delta y_{2}(a)\right)^{\alpha_{*}}+\sum_{a}^{n-1} p_{2}(s)\left(y_{2}(s)\right)^{\beta_{*}}\right)^{1 / \alpha^{*}}\right]<0 .
\end{aligned}
$$

Hence we see that $y_{1}(n)<y_{2}(n)$ for $a<n \leq b$. Returning to (20), we find that $\Delta y_{1}(n)<\Delta y_{2}(n)$ for $a \leq n \leq b$. The proof is complete. 
The uniqueness of local solutions with non-zero initial data can be easily proved. That is, for given $N\left(\geq n_{0}\right)$, $y_{0}$ and $y_{1}$, Equation (8) has a unique local solution $y(n)$ satisfying $y(N)=y_{0}, \Delta y(N)=y_{1}$ provided that $\left|y_{0}\right|+\left|y_{1}\right| \neq 0$. The uniqueness of the trivial solution can be concluded for the case $\alpha \leq \beta$.

Lemma 3. Let $\alpha \leq \beta$ and $N \geq n_{0}$. If $y(n)$ is a local solution of Equation (1) satisfying $y(N)=\Delta y(N)=0$ then $y(n) \equiv 0$ for $n_{0} \leq n<\infty$.

Proof. Assume the contrary. We may suppose that $y(n) \neq \equiv$ for $N \leq n<\infty$. Then, we can find $n_{1}, n_{2}$ such that $N \leq n_{1}<n_{2}$ satisfying $\left|y\left(n_{1}\right)\right|+\left|\Delta y\left(n_{1}\right)\right|=0$ and $|y(n)|+|\Delta y(n)|>0$ for $n_{1}<n \leq n_{2}$. Summing (8), we obtain

$$
\begin{aligned}
& \Delta y(n)=\left(\sum_{n_{1}}^{n-1} p(s)(y(s))^{\beta_{*}}\right)^{1 / \alpha^{*}}, \\
& y(n)=\sum_{n_{1}}^{n-1}\left(\sum_{n_{1}}^{s-1} p(r)(y(r))^{\beta_{*}}\right)^{1 / \alpha^{*}}, n_{1} \leq n \leq n_{2} .
\end{aligned}
$$

We therefore have

$$
\begin{aligned}
& |\Delta y(n)| \leq\left(\sum_{n_{1}}^{n-1} p(s)\{|y(s)|+|\Delta y(s)|\}^{\beta}\right)^{1 / \alpha} \\
& |y(n)| \leq \sum_{n_{1}}^{n-1}\left(\sum_{n_{1}}^{s-1} p(r)\{|y(r)|+|\Delta y(r)|\}^{\beta}\right)^{1 / \alpha}, n_{1} \leq n \leq n_{2} .
\end{aligned}
$$

Put $w(n)=\max _{n_{1} \leq \xi \leq n}(|y(\xi)|+|\Delta y(\xi)|)$. We see that $w\left(n_{1}\right)=0, w(n)>0$ for $n_{1}<n \leq n_{2}$ and $w$ is nondecreasing. From (22) and (23), we can get

$$
\begin{aligned}
& |\Delta y(n)| \leq(w(n))^{\beta / \alpha}\left(\sum_{n_{1}}^{n-1} p(s)\right)^{1 / \alpha}, \\
& |y(n)| \leq(w(n))^{\beta / \alpha} \sum_{n_{1}}^{n-1}\left(\sum_{n_{1}}^{s-1} p(r)\right)^{1 / \alpha}, n_{1} \leq n \leq n_{2} .
\end{aligned}
$$

Let $n_{1} \leq \tau \leq n \leq n_{2}$. Then from this observation we see that

$$
|\Delta y(\tau)|+|y(\tau)| \leq(w(\tau))^{\beta / \alpha} G(\tau) \leq(w(n))^{\beta / \alpha} G(n),
$$

where

$$
G(v)=\left(\sum_{n_{1}}^{v-1} p(s)\right)^{1 / \alpha}+\sum_{n_{1}}^{v-1}\left(\sum_{n_{1}}^{s-1} p(r)\right)^{1 / \alpha}
$$

Consequently, we have

$$
w(n) \leq(w(n))^{\beta / \alpha} G(n), n_{1} \leq n \leq n_{2} .
$$

If $\alpha=\beta$, from (24), we have $1 \leq G(n), n_{1}<n \leq n_{2}$. This is a contradiction because $G\left(n_{1}\right)=0$. If $\alpha<\beta$, from (24) we have $(w(n))^{-\frac{\beta-\alpha}{\alpha}} \leq G(n), n_{1}<n \leq n_{2}$. This is also a contraction because $G\left(n_{1}+0\right)=w\left(n_{1}+0\right)=0$. The proof is complete.

Lemma 4. Let $\alpha \geq \beta$. Then all local solutions of Equation (8) can be continued to $\infty$ and $n_{0}$, that is, all solutions of Equation (8) exist on the whole interval $\left[n_{0}, \infty\right)$.

Proof. Let $y(n)$ be a local solution of Equation (8) is a neighborhood of $N \geq n_{0}$. Suppose the contrary that the right maximal interval of existence of $y(n)$ is of the form $[N, w), w<\infty$. Then, it is easily seen that $y(w-0)= \pm \infty$. Summing (8) twice, we have 


$$
y(n)=c_{0}+\sum_{N}^{n-1}\left(c_{1}^{\alpha_{*}}+\sum_{N}^{s-1} p(r)(y(r))^{\beta_{*}}\right)^{1 / \alpha^{*}}
$$

where $c_{0}=y(N)$ and $c_{1}=\Delta y(N)$. Accordingly,

$$
|y(n)| \leq\left|c_{0}\right|+\sum_{N}^{n-1}\left(\left|c_{1}\right|^{\alpha}+\sum_{N}^{s-1} p(r)|y(r)|^{\beta}\right)^{1 / \alpha}, N \leq n<w .
$$

Put $z(n)=\max _{N \leq \xi \leq n}|y(\xi)|$. Then,

$$
|y(n)| \leq\left|c_{0}\right|+\sum_{N}^{n-1}\left(\left|c_{1}\right|^{\alpha}+|z(s)|^{\beta} \sum_{N}^{s-1} p(r)\right)^{1 / \alpha}, N \leq n<w .
$$

Put moreover $u(n)=\max \left\{\left|c_{1}\right|^{\alpha / \beta}, z(n)\right\}$. Then, as in the proof of Lemma 3, we have

$$
|z(n)| \leq\left|c_{0}\right|+\sum_{N}^{n-1} H(s)(u(s))^{\beta / \alpha}, N \leq n<w
$$

where $H(n)=\left(1+\sum_{N}^{n-1} p(s)\right)^{1 / \alpha}$. Since $y(w-0)= \pm \infty$, there is a $\bar{N}$ such that $N<\bar{N}<w$ such that $z(n) \geq\left|c_{1}\right|^{\alpha / \beta}$ for $\bar{N} \leq n<w$. Therefore it follows from (25) that

$$
u(n) \leq\left|c_{0}\right|+\sum_{N}^{n-1} H(s)(u(s))^{\beta / \alpha}, \bar{N} \leq n<w .
$$

Let $\alpha=\beta$. Then, using discrete Gronwall's inequality, we see that $u(w-0)<\infty$, which is a contradiction. Next let $\alpha>\beta$. Then (26) implies that

$$
u(n) \leq\left|c_{0}\right|+(u(n))^{\beta / \alpha} \sum_{N}^{n-1} H(s), \quad \bar{N} \leq n<w .
$$

Since $\beta / \alpha<1$, we have $u(w-0)<\infty$. This is a contradiction too. Hence $y(n)$ can be continued to $\infty$. The continuability to the left end point $n_{0}$ is verified in a similar way. The proof is complete.

The following lemma establishes more than is stated in Theorem 8. Accordingly the proof of Theorem 8 will be omitted.

Lemma 5. Let $\alpha<\beta$ and $N \geq n_{0}$ and $c>0$ be given. Then there exists an $M=M(N, c)>0$ such that the right maximal interval of existence of each solution $y(n)$ of Equation (1) satisfying $y(N) \geq c$ and $\Delta y(N) \geq M$ is a finite interval $[N, \bar{N}), \bar{N}=\bar{N}_{y(n)}<\infty$, and $\lim _{n \rightarrow N-0} y(n)=\infty$.

Proof. Let $n_{1}>N$ be fixed, and put $\min _{N \leq n \leq n_{1}} p(n)=m>0$. There is an $M>0$ satisfying

$$
\sum_{c}^{\infty}\left(M^{\alpha}+m\left((v(n))^{\beta+1}-c^{\beta+1}\right)\right)^{-\frac{1}{\alpha}}<n_{1}-N .
$$

We first claim that the solution of Equation (8) with the initial condition $z(n)=c, \Delta z(n)=M$ does not exist on $\left[N, n_{1}\right)$; that is $z(n)$ blow up at some $\bar{N} \in\left(N, n_{1}\right]$. To see this suppose the contrary that $z(n)$ exists at least $\left[N, n_{1}\right)$. By the definition of $m$, we have

$$
\Delta(\Delta z(n))^{\alpha}=p(n)(z(n))^{\beta} \geq m(z(n))^{\beta}, \quad N \leq n \leq n_{1} .
$$

Summing the inequality form $N$ to $n-1$ yields

$$
(\Delta z(n))^{\alpha}-M^{\alpha} \geq m\left((z(n+1))^{\beta}-C^{\beta}\right), \quad N \leq n \leq n_{1}
$$

and hence 


$$
\begin{aligned}
& (\Delta z(n))^{\alpha} \geq M^{\alpha}+m\left(z(n)^{\beta}-C^{\beta}\right) \\
& \Delta z(n) \geq\left(M^{\alpha}+m\left((z(n))^{\beta}-C^{\beta}\right)\right)^{\frac{1}{\alpha}} \\
& \Delta z(n)\left[M^{\alpha}+m\left((z(n))^{\beta}-C^{\beta}\right)\right]^{-\frac{1}{\alpha}} \geq 1, \quad N \leq n \leq n_{1} .
\end{aligned}
$$

Finally, summing the above inequality both sides from $N$ to $n_{1}-1$, we obtain

$$
\sum_{n}^{z\left(n_{1}\right)}\left[M^{\alpha}+m\left((w(n))^{\beta}-C^{\beta}\right)\right]^{-\frac{1}{\alpha}} \geq n_{1}-N,
$$

which is a contradiction to the choice of $M$. Hence $z(n)$ must blow up at some $\bar{N} \in\left(N, n_{1}\right], \lim _{n \rightarrow \bar{N}-0} z(n)=\infty$.

If $y(N) \geq c$ and $\Delta y(N) \geq M$, then Lemma 2 implies that $y(n) \geq z(n)$ on the common interval of existence of $y$ and $z$ and therefore $y(n)$ blows up at some point before $n_{1}$. The proof is complete.

\section{Nonnegative Nonincreasing Solutions}

The main objective of this section is to prove the following theorem.

Theorem 17. For each $y_{0}>0$, the problem

$$
\left\{\begin{array}{l}
\Delta\left(|\Delta y(n)|^{\alpha-1} \Delta y(n)\right)=p(n)|y(n)|^{\beta-1} y(n) \\
y\left(n_{0}\right)=y_{0}
\end{array}\right.
$$

has exactly one solution $\bar{y}$ such that $\bar{y}$ is defined for $n \geq n_{0}$ and satisfies

$$
\bar{y}(n) \geq 0, \quad \Delta y(n) \leq 0 \text { for } n \geq n_{0} .
$$

Furthermore, if $y(n)$ is a solution for $n \geq n_{0}$ of Equation (1) satisfying $y\left(n_{0}\right)=y_{0}$ and

$$
\Delta y\left(n_{0}\right)>\Delta \bar{y}\left(n_{0}\right)\left[\operatorname{resp} \Delta y\left(n_{0}\right)<\Delta \bar{y}\left(n_{0}\right)\right],
$$

then

$$
\lim _{n \rightarrow \infty} y(n)=\infty \quad\left[\operatorname{resp} \lim _{n \rightarrow \infty} y(n)=-\infty\right]
$$

\section{Remark 4.}

1) In the case $\alpha \leq \beta$, employing Lemma 3, we can strengthen (27) to the property that

$$
\Delta y(n)>0, \Delta \bar{y}(n)<0 \text { for } n \geq n_{0} .
$$

2) In the case $\alpha \geq \beta$, all local solutions of Equation (8) can be continued to the whole interval $\left[n_{0}, \infty\right)$ Hence in this case property (6.2) always holds for all solutions $y(n)$ with $y\left(n_{0}\right)=y_{0}$ and $\Delta y\left(n_{0}\right)>\Delta \bar{y}\left(n_{0}\right)$ [resp $\left.\Delta y\left(n_{0}\right)<\Delta \bar{y}\left(n_{0}\right)\right]$.

The property of nonnegative nonincreasing solutions $\bar{y}$ described in Theorem 17 will play important roles through the paper. This section is entirely derided to proving Theorem 17. To this end we prepare several lemmas.

Lemma 6. Let $A, B \in R$ and $t$ be a bounded function on $[a, b] \times R$. Then, the two point boundary value problem

$$
\left\{\begin{array}{l}
\Delta\left(|\Delta y(n)|^{\alpha-1} \Delta y(n)\right)=f(n, y(n)), \quad a \leq n \leq b, \\
y(a)=A, \quad y(b)=B,
\end{array}\right.
$$

has a solution.

Proof. Let $K>0$ be a constant such that 


$$
|f(n, y(n))| \leq K, \quad \text { for }(n, y(n)) \in[a, b] \times R .
$$

We first claim that with each $y(n)$, we can associate a unique constant $c(y)$ satisfying

$$
\sum_{a}^{b}\left(c(y)+\sum_{a}^{s} f(r, y(r))\right)^{1 / \alpha^{*}}=B-A .
$$

Further this $c(y)$ satisfies

$$
-K(b-a)+\left(\frac{B-A}{b-a}\right)^{\alpha^{*}} \leq c(y) \leq K(b-a)+\left(\frac{B-A}{b-a}\right)^{\alpha^{*}} .
$$

To see this let $y(n)$ be fixed, and consider the function

$$
I(\lambda)=\sum_{a}^{b}\left(\lambda+\sum_{a}^{s} f(r, y(r))\right)^{1 / \alpha^{*}}, \lambda \in R .
$$

If $\lambda<-K(b-a)+\left(\frac{B-A}{b-a}\right)^{\alpha_{*}}$, then $I(\lambda)<B-A$. If $\lambda>K(b-a)+\left(\frac{B-A}{b-a}\right)^{\alpha_{*}}$, then $I(\lambda)>B-A$. Since $I$ is a strictly increasing continuous function, there is a unique constant $c(y)$ satisfying $I(c(y))=B-A$, namely (30). Then (31) is clearly satisfied.

By (31), we see that there is a constant $M=M(a, b, A, B, K)>0$ satisfying $|c(y)| \leq M$ for all $y(n)$. Choose $L>0$ so large that

$$
|A| \leq L \text { and } M+K(b-a)^{1 / \alpha}(b-a) \leq L .
$$

Consider the Banach space $B_{N}$ of all real sequences $y=\{y(n)\}_{n \geq N}$ with the supernum norm $\|y\|=\sup _{n \geq N}|y(n)|$. Now we define the set $y \subset B_{N}$ and the mapping $F: Y \rightarrow B_{N}$ by

$$
Y=\left[y(n) \in B_{N_{0}}:|y(n)| \leq 2 L \text { for } a \leq n \leq b\right]
$$

and

$$
F(y(n))=A+\sum_{a}^{n}\left(c(y)+\sum_{a}^{s} f(r, y(r))\right)^{1 / \alpha^{*}}, a \leq n \leq b
$$

respectively. Then the boundary value problem (29) is equivalent to finding a fixed element of $\mathcal{F}$. We show that $F$ has a fixed element in $Y$ (via) the Schavder fixed point theorem

$$
\begin{aligned}
|F(y(n))| & \leq|A|+\sum_{a}^{n}\left(|c(y)|+\sum_{a}^{s}|f(r, y(r))|\right)^{1 / \alpha} \\
& \leq|A|+\sum_{a}^{n} M+K(s-a)^{1 / \alpha} \\
& \leq|A|+M(b-a)^{1 / \alpha}(b-a) \\
& \leq L+L=2 L, \quad a \leq n \leq b .
\end{aligned}
$$

Hence $F$ maps $Y$ into itself.

Next, to see the continuity of $F$, assume that $y_{k}(n)$ be a sequence converging to $y \in Y$ uniformly in $[a, b]$. We must prove that $F\left(y_{k}(n)\right)$ converges to $F y_{k}(n)$ uniformly in $[a, b]$. As a first step, we show that $\lim _{n \rightarrow \infty} c\left(y_{k}(n)\right)=c(y(n))$. Assume that this is not the case. Then because of the boundedness of $\left\{c\left(y_{k}(n)\right)\right\}$, there is a subsequence $\left\{c\left(y_{k_{i}}(n)\right)\right\}$ satisfying $c y_{k_{i}}(n) \rightarrow \bar{c} \neq c(y)$ for some finite value $\bar{c}$. Noting the relation

$$
\sum_{a}^{b}\left(c\left(y_{k_{i}}(n)\right)+\sum_{a}^{s} f\left(r, y_{k_{i}}(r)\right)\right)^{1 / \alpha_{*}}=B-A .
$$


We have

$$
\begin{aligned}
B-A & =\lim _{K_{i} \rightarrow \infty} \sum_{a}^{b}\left(c\left(y_{k_{i}}(n)\right)+\sum_{a}^{s} f\left(r, y_{k_{i}}(r)\right)\right) \\
& =\sum_{a}^{b}\left(\bar{c}+\sum_{a}^{b} f(r, y(r))\right)^{1 / \alpha^{*}} .
\end{aligned}
$$

This contradicts the uniqueness of the number $c(y)$. Hence $\lim _{n \rightarrow \infty} c(y(n))=c(y)$. Then we find similarly that $\lim _{n \rightarrow \infty} F\left(y_{k}(n)\right)=F(y(n))$ uniformly on $[a, b]$.

It will be easily seen that the sets

$$
F Y=\{F y: y \in Y\} \text { and }\{\Lambda(F(y)): y \in Y\}
$$

are uniformly bounded on $[a, b]$. Then $F \bar{Y}$ is compact.

From the above observations we see that $F$ has a fixed element in $Y$. Then this fixed element is a solution of boundary value problem (29) is easily proved. The proof is now complete.

Lemma 7. Let $n_{1}>n_{0}$ and $n_{0}>0$. Then the two point boundary value problem

$$
\left\{\begin{array}{l}
\Delta\left(|\Delta y(n)|^{\alpha-1} \Delta y(n)\right)=p(n)|y(n)|^{\beta-1} y(n) \text { for } n_{0} \leq n \leq n_{1} \\
y\left(n_{0}\right)=y_{0}, \quad y\left(n_{1}\right)=0
\end{array}\right.
$$

has a solution $y(n)$ such that $y(n) \geq 0$ and $\Delta y(n) \leq 0$ for $n_{0} \leq n \leq n_{1}$.

Proof. Define the bounded function $f$ on $\left[n_{0}, n_{1}\right] \times R$ by

$$
f(n, y(n))= \begin{cases}p(n) y_{0}^{\beta} & \text { for } n_{0} \leq n \leq n_{1}, \quad y \geq y_{0} \\ p(n) y^{\beta} & \text { for } n_{0} \leq n \leq n_{1}, \quad 0 \leq y \leq y_{0} \\ 0 & \text { for } n_{0} \leq n \leq n_{1}, \quad y \leq 0 .\end{cases}
$$

By Lemma 6, the boundary value problem

$$
\left\{\begin{array}{l}
\Delta\left(|\Delta y(n)|^{\alpha-1} \Delta y(n)\right)=f(n, y(n)), \quad n_{0} \leq n \leq n_{1} \\
y\left(n_{0}\right)=y_{0}, \quad y\left(n_{1}\right)=0
\end{array}\right.
$$

has a solution $y$.

We show that $y$ satisfies $y(n) \geq 0$ for $n_{0} \leq n \leq n_{1}$. If this is not the case, we can find an interval $\left[\tau_{0}, \tau_{1}\right] \subset\left[n_{0}, n_{1}\right]$ such that $y(n)<0$ on $\left[\tau_{0}, \tau_{1}\right]$ and $y\left(\tau_{0}\right)=y\left(\tau_{1}\right)=0$. The definition of $f$ implies that $y$ satisfies the equation $\Delta\left(|\Delta y(n)|^{\alpha-1} \Delta y(n)\right)=0$ on $\tau_{0} \leq n \leq \tau_{1}$. Hence $y(n)$ is a linear function on $\left[\tau_{0}, \tau_{1}\right]$. Obviously that this is a contradiction. We see therefore that $y(n) \geq 0$ on $\left[n_{0}, n_{1}\right]$.

Since $\Delta y\left(n_{1}\right) \leq 0$ and $\Delta\left(|\Delta y(n)|^{\alpha-1} \Delta y(n)\right) \geq 0$ on $\left[n_{0}, n_{1}\right]$, by the definition of $t$, we find that $\Delta y(n) \leq 0$ on $\left[n_{0}, n_{1}\right]$. Hence $y(n) \leq y_{0}$, which implies that $y$ is a desired solution of problem (32). The proof is complete.

Proof of Theorem 17. The uniqueness of $\bar{y}$ satisfying the properties mentored here is easily established as in the proof Lemma 2 . Therefore we prove only the existence of such a $\bar{y}$.

By Lemma 7, for each $k \in N$, we have a solution $y=y_{0}$ of the boundary value problem

$$
\left\{\begin{array}{l}
\Delta\left(|\Delta y(n)|^{\alpha-1} \Delta y(n)\right)=p(n)|y(n)|^{\beta-1} y(n) \\
y\left(n_{0}\right)=y_{0}, \quad y\left(n_{0}+k\right)=0, \text { for } n_{0} \leq n \leq n_{0}+k
\end{array}\right.
$$

satisfying $y_{k}(n) \geq 0$ and $\Delta y_{k}(n) \leq 0$ for $n_{0} \leq n \leq n_{0}+k$ let us extend each $y_{k}$ over the interval $\left[n_{0}, \infty\right)$ 
by defining $y_{k} \equiv 0$ for $n \geq n_{0}+k$. Below we show that $\left\{y_{k}(n)\right\}$ contains a subsequence converging to a desired solution of (8).

As a first step, we prove that

$$
\Delta y_{1}\left(n_{0}\right) \leq \Delta y_{2}\left(n_{0}\right) \leq \cdots \leq y_{k}\left(n_{0}\right) \leq 0 .
$$

In fact, if this is not case, then $\Delta y_{i}\left(n_{0}\right)>\Delta y_{i+1}\left(n_{0}\right)$ for some $i$. Since $y_{i}\left(n_{0}\right)=y_{i+1}\left(n_{0}\right)$. Lemma 2 implies that $y_{i}(n)>y_{i+1}(n)$ for $n_{0} \leq n \leq n_{0}+i$. Putting $n=n_{0}+i$, we have $0=y_{i}\left(n_{0}+i\right)>y_{i+1}\left(n_{0}+i\right) \geq 0$ a contradiction. Accordingly (33) holds, and so $\lim _{n \rightarrow \infty} \Delta y_{k}\left(n_{0}\right)=l \in(-\infty, 0]$ exists, since $0 \leq y_{k}(n) \leq y_{0}$ on $\left[n_{0}, n_{0}+k\right]$ for any $k \in N,\left\{y_{k}(n)\right\}$ is uniformly bounded on each compact subinterval of $\left[n_{0}, \infty\right)$. Noting that $\Delta y_{k}(n)$ is nondecreasing and nonpositive on $\left[n_{0}, n_{0}+k\right]$, we have

$$
\Delta y_{1}\left(n_{0}\right) \leq \Delta y_{k}\left(n_{0}\right) \leq \Delta y_{k}(n) \leq 0 \quad \text { on }\left[n_{0}, n_{0}+k\right], k \in N .
$$

Hence $\left\{y_{k}(n)\right\}$ is equicontinuous on each compact subinterval of $\left[n_{0}, \infty\right)$. From these consideration we find that there is a subsequence $\left\{y_{k_{i}}(n)\right\} \subset\left\{y_{k}(n)\right\}$ and a function $\bar{y}$ satisfying $\lim _{k_{i} \rightarrow \infty}\left(y_{k_{i}}(n)\right)=\bar{y}(n)$ uniformly on each compact subinterval of $\left[n_{0}, \infty\right)$. Finally we shall show that $\bar{y}$ is a desired solution of Equation (8). Let $n \in\left[n_{0}, \infty\right)$ be fixed arbitrarily. For all sufficiently large $k_{i}$ 's we have

$$
y_{k_{i}}(n)=y_{0}+\sum_{n_{0}}^{n-1}\left(\left|\Delta y_{k_{i}}\left(n_{0}\right)\right|^{\alpha_{*}}+\sum_{n_{0}}^{s} p(r)\left|y_{k_{i}}(r)\right|^{\beta}\right)^{1 / \alpha^{*}}
$$

letting $k_{i} \rightarrow \infty$, we obtain

$$
\bar{y}(n)=y_{0}+\sum_{n_{0}}^{n-1}\left(l^{\alpha_{*}}+\sum_{n_{0}}^{s} p(r)[\bar{y}(r)]^{\beta}\right)^{1 / \alpha^{*}} .
$$

Taking difference in this above equality, we are that $\bar{y}$ solves Equation (8) on $\left[n_{0}, \infty\right)$. That $\bar{y}$ satisfies (27) is evident. The proof of Theorem 17 is complete.

\section{Proofs of Main Results for the Super-Homogeneous Equations}

Throughout this section, we assume that $\alpha<\beta$.

Proof of Theorem 2. The theorem is an immediate consequence of the uniqueness of the trivial solution (Lemma 3).

Proof of Theorem 4. Necessity Part: Let $y(n)$ be a positive solution of Equation (8) for $n \geq n_{1}$ of type (AC). It is easy to see that $\Delta y(n) \uparrow 0$ and $y(n) \downarrow y(\infty)>0$ as $n \uparrow \infty$. Hence summing (8) twice, we have

$$
-y(\infty)+y\left(n_{1}\right)=\sum_{n_{1}}^{\infty}\left(\sum_{s}^{\infty} p(r)|y(r)|^{\beta}\right)^{1 / \alpha}
$$

from which we find that

$$
(y(\infty))^{\beta / \alpha} \sum_{n_{1}}^{\infty}\left(\sum_{s}^{\infty} p(r)\right)^{1 / \alpha}<\infty .
$$

This is equivalent to (10).

Sufficiency Part: Let (10) hold. Fix an $l>0$ and choose $n_{1} \geq n_{0}$ so that

$$
\sum_{n_{1}}^{\infty}\left(\sum_{s}^{\infty} p(r)\right)^{1 / \alpha} \leq \frac{(2 l)^{\frac{\alpha-\beta}{\alpha}}}{2} .
$$

We introduce the Banach space $I^{\infty}$ of all bounded, real sequences $\{y(n)\}$ with norm $\|y\|=\sup _{n}|y(n)|$. Define the set $Y=\left\{y(n) \in l^{\infty}: l \leq y(n) \leq 2 l, n \geq n_{1}\right\}$ and the mapping $F: Y-l^{\infty}$ by 


$$
F y(n)=l+\sum_{n}^{\infty}\left(\sum_{s}^{\infty} p(r)|y(r)|^{\beta}\right)^{1 / \alpha}, n \geq n_{1} .
$$

We below show via the Schauder-Tychonoff fixed point theorem that $F$ has at least one fixed element in $Y$. Firstly, let $y(n) \in Y$. Then

$$
l \leq F y(n) \leq l+(2 l)^{\beta / \alpha} \sum_{n_{1}}^{\infty}\left(\sum_{s}^{\infty} p(r)\right)^{1 / \alpha} \leq l+l=2 l, n \geq n_{1} .
$$

Thus $F y \in Y$, and hence $F Y \subset Y$. Secondly, to see the continuity of $F$, let $\left\{y_{k}(n)\right\}$ be a sequence in $Y$ covering to $y(n) \in Y$ uniformly on each compact subinterval of $\left[n_{1}, \infty\right)$ since $p(n)$ is bounded for $n_{1} \leq n \leq \infty$ and

$$
0 \leq \sum_{s}^{\infty} p(r)\left[y_{k}(r)\right]^{\beta} \leq(2 l)^{\beta} \sum_{s}^{\infty} p(r)<\infty .
$$

The Lebesgue dominated convergence theorem implies that $F y_{k}(n) \rightarrow F y(n)$ uniformly on each compact subinterval of $\left[n_{1}, \infty\right)$ since for $y(n) \in Y$,

$$
|\Delta(F y(n))| \leq\left(\sum_{n}^{\infty} p(s)[y(s)]^{\beta}\right)^{1 / \alpha} \leq(2 l)^{\beta / \alpha}\left(\sum_{n_{1}}^{\infty} p(s)\right)^{1 / \alpha}, n \geq n_{1} .
$$

The set $\{\Delta(F y(n)): y \in Y\}$ is uniformly bounded on $\left[n_{1}, \infty\right)$. This implies that $\bar{F}_{Y}$ is compact.

From there observations we find that $F$ has a proved element $y$ in $Y$ such that $F y=y$. That this $y$ is a solution of Equation (1) of type (AC) is easily proved. The proof is complete.

Proof of Theorem 3. Sufficiency Part: Let $\bar{y}(n)$ be a solution of Equation (8) satisfying $\bar{y}(n)>0$, $\Delta \bar{y}(n)<0$ for $n \geq n_{0}$. The existence of such a solution is ensured by Theorem 17. Obviously, $\bar{y}(n)$ is either of type $(D)$ or type $(A C)$. Theorem 4 shows that under assumption (9), Equation (8) does not posses solutions of type (AC). Hence $\bar{y}(n)$ must be of type $(D)$.

Necessity Part: Let $y(n)$ be a positive solution of Equation (8) for $n \geq n_{1}$ of type $(D)$. Clearly $y(n)$ satisfies

$$
y(n)=\sum_{n}^{\infty}\left(\sum_{s}^{\infty} p(r)|y(r)|^{\beta}\right)^{1 / \alpha}, n \geq n_{1} .
$$

To verify (9), suppose the contrary that (9) fails to hold. Then, nothing that $y(n)$ is decreasing for $n \geq n_{1}$, we have

$$
y(n) \leq|y(n)|^{\beta / \alpha} \sum_{n}^{\infty}\left(\sum_{s}^{\infty} p(r)\right)^{1 / \alpha}, \quad n \geq n_{1} .
$$

Accordingly,

$$
|y(n)|^{1-\beta / \alpha} \leq \sum_{n}^{\infty}\left(\sum_{s}^{\infty} p(r)\right)^{1 / \alpha}, \quad n \geq n_{1} .
$$

The left hand side tends to $\infty$ as $n \rightarrow \infty$ because of $\alpha<\beta$, where as the right hand side tends to 0 as $n \rightarrow \infty$. This contradiction verifies (9). The proof is complete.

Proof of Theorem 5. Necessity Part: Let $y(n)$ be a positive solution of Equation (8) near $\infty$ of type $(A L)$. There is a constant $c>0$ and $n_{1} \geq n_{0}$ satisfying

$$
y(n) \geq c n, \quad n \geq n_{1} .
$$

Summation of Equation (8) from $n_{1}$ to $n-1$ yields

$$
[\Delta y(n)]^{\alpha_{*}}-\left[\Delta y\left(n_{1}\right)\right]^{\alpha_{*}}=\sum_{n_{1}}^{n-1} p(s)(y(s))^{\beta}, \quad n \geq n_{1} .
$$


Since $\lim _{n \rightarrow \infty} \Delta y(n)=\Delta y(\infty) \in(0, \infty)$, this in equality implies that

$$
\sum_{n_{1}}^{\infty} p(n)[y(n)]^{\beta}<\infty .
$$

Combining (35) with (34), we find that (11) holds.

Sufficiency Part: We fix $l>0$ arbitrarily, and choose $n_{1} \geq n_{0}$ large enough so that

$$
\sum_{n_{1}}^{\infty} n^{\beta} p(n) \leq(2 l)^{\alpha-\beta}\left(1-\frac{1}{2^{\alpha}}\right) .
$$

Let $l^{\alpha}$ be the Banach space as in the proof Theorem 4. Define the set $Y \in l^{\infty}$ as follows

$$
Y=\left\{y(n) \in l^{\infty}: l\left(n-n_{1}\right) \leq y(n) \leq 2 l\left(n-n_{1}\right), n \geq n_{1}\right\} .
$$

The mapping $F: Y \rightarrow l^{\infty}$ defined by

$$
F y(n)=\sum_{n_{1}}^{n-1}\left((2 l)^{\alpha}-\sum_{s}^{\infty} p(r)[y(r)]^{\beta}\right)^{1 / \alpha}, \quad n \geq n_{1} .
$$

As in the proof of the sufficiency part of Theorem 4, we can show that $F$ has a fixed element $y(n) \in Y$ by the Schavder-Tyehonoff fixed point Theorem

$$
y(n)=\sum_{n_{1}}^{n-1}\left((2 l)^{\alpha}-\sum_{s}^{\infty} p(r)[y(r)]^{\beta}\right)^{1 / \alpha}, \quad n \geq n_{1} .
$$

Taking $\Delta$ twice for this formula we see that $y(n)$ is a positive solution of Equation (8) for $n \geq n_{1}$. L'Hospital's rule shows that $\lim _{n \rightarrow \infty} \frac{y(n)}{n}=2 l$. Thus $y(n)$ is a solution of Equation (8) of type $(A L)$. The proof is complete.

Lemma 8. Let $y(0)>0$. If (11) holds, then there is a positive solution of Equation (8) for $n \geq n_{0}$ of type $(A L)$ satisfying $y\left(n_{0}\right)=y(0)$.

Proof. By Theorem 5, there is an (AL)-type positive solution $z(n)$ of Equation (8) defined in some neighborhood of $\infty: 0<\lim _{n \rightarrow \infty} \frac{z(n)}{n}=\lim _{n \rightarrow \infty} \Delta z(n)<\infty$. Let $\bar{y}(n)$ be a positive solution of Equation (8) for $n \geq n_{0}$ satisfying $\bar{y}\left(n_{0}\right)=y(0)$ and $\bar{y}(n)>0, \Delta \bar{y}(n)<0$ for $n \geq n_{0}$. Take a $n_{1}>n_{0}$ such that $\bar{y}(n)<z(n)$ and $\Delta \bar{y}(n)<\Delta z(n)$ for $n \geq n_{1}$. By Lemma 2 if $\lambda>\Delta \bar{y}\left(n_{0}\right)$ is sufficiently elver to $\Delta \bar{y}\left(n_{0}\right)$, then the solution $y(n)$ of Equation (8) with $y\left(n_{0}\right)=y(0)$ and $\Delta y\left(n_{0}\right)=\lambda$ exists at least on $\left[n_{0}, n_{1}\right]$ and satisfies

$$
\bar{y}\left(n_{1}\right)<y\left(n_{1}\right)<z\left(n_{1}\right), \quad \Delta \bar{y}\left(n_{1}\right)<\Delta y\left(n_{1}\right)<\Delta z\left(n_{1}\right) .
$$

Then Lemma 2 again implies that $\bar{y}(n)<y(n)<z(n)$ as long as $y(n)$ exists. Since $\bar{y}(n)$ and $z(n)$ exists for $n \geq n_{1}$, this means that $y(n)$ exists for $n \geq n_{1}$ and satisfies $\bar{y}(n)<y(n)<z(n), n \geq n_{1}$. Then we have

$$
\frac{\bar{y}(n)}{n}<\frac{y(n)}{n}<\frac{z(n)}{n}, \quad n \geq n_{1} .
$$

Noting that $\bar{y}(n)$ is the unique solution of (8) satisfying $\lim _{n \rightarrow \infty} \frac{\bar{y}(n)}{n}=0$ and passing through the point $\left(n_{0}, y(0)\right)$ we have $\lim \lim _{n \rightarrow \infty} \frac{y(n)}{n} \in(0, \infty)$. Therefore $y(n)$ is of type $(A L)$. The proof is complete.

Proof of Theorem 6. For $\lambda>0$, we denote by $y_{\lambda}(n)$, the unique solution of Equation (8) with in initial condition $y\left(n_{0}\right)=y(0)$ and $\Delta y\left(n_{0}\right)=\lambda$. The maximal interval of existence of $y_{k}(n)$ may be finite or infinite.

Define the set $S \subset(0, \infty)$ by 


$$
S=\left\{\lambda>0: y_{\lambda}(n) \text { exists for } n \geq n_{0} \text { and is of type }(A L)\right\}
$$

We know by Lemma 8 that $S \neq Q$ and by Lemma 5 that $\lambda \notin S$ for all sufficiently large $\lambda>0$. Hence $\sup S=\bar{\lambda} \in(0, \infty)$ exists. For $\bar{\lambda}$ there are three possibilities:

1) $\bar{\lambda} \in S$

2) $\bar{\lambda} \notin S$ and $y_{\bar{\lambda}}(n)$ is of type (AS)

3) $\bar{\lambda} \notin S$ and $\lambda_{\bar{\lambda}}(n)$ is of type $\left(S_{2}\right)$.

To prove the theorem, we below show that case (b) occurs. For simplicity, we write $\bar{y}$ for $y_{\bar{\lambda}}$ below. Suppose that the case (a) occurs. Then $\lim _{n \rightarrow \infty} \Delta \bar{y}(n)=\Delta \bar{y}(\infty)=l \in(0, \infty)$ and $\Delta \bar{y}(n)<l, n \geq n_{0}$. By condition (11) we can find a $n_{1}>n_{0}$ satisfying

$$
\sum_{n_{0}}^{\infty} p(s)\left(y_{0}+2 l s\right)^{\beta}<(2 l)^{\alpha}-l^{\alpha}
$$

Choose $\lambda>\bar{\lambda}$ close enough to $\bar{\lambda}$ so that $y_{\lambda}(n)$ exists at least on $\left[n_{0}, n_{1}\right]$ and $\Delta y_{\lambda}\left(n_{1}\right)<l$. Then, for such a $\lambda, y_{\lambda}(n)$ can be extended to $\infty$, and satisfies $\Delta y_{\lambda}(n)<2 l, n \geq n_{1}$. In fact, if this is not the case, there is $\bar{n}>n_{1}$ satisfying $\Delta y_{\lambda}(n)<2 l$ for $n_{0} \leq n<\bar{n}$ and $\Delta y_{\lambda}(\bar{n})=2 l$. It follows therefore that $y_{\lambda}(n) \leq y(0)+2 l$ for $n_{0} \leq n \leq \bar{n}$. Summing the Equation (8) (with $y=y_{\lambda}$ ) for $n_{1} \leq n \leq \bar{n}$ yields

$$
\begin{aligned}
(2 l)^{\alpha} & =\left(\Delta y_{\lambda}(\bar{n})\right)^{\alpha}=\left(\Delta y_{\lambda}\left(n_{1}\right)\right)^{\alpha}+\sum_{n_{1}}^{\bar{n}-1} p(s)\left|y_{\lambda(s)}\right|^{\beta} \\
& \leq l^{\alpha}+\sum_{n_{1}}^{\bar{n}-1} p(s)(y(0)+2 l s)^{\beta} \leq l^{\alpha}+\sum_{n_{1}}^{\infty} p(s)(y(0)+2 l s)^{\beta}<(2 l)^{\alpha} .
\end{aligned}
$$

This contradiction implies that $y_{\lambda}(n)$ exists for $n \geq n_{0}$ and satisfies $\Delta y_{\lambda}(n)<2 l, n \geq n_{0}$. These observations show that $S \ni \lambda>\bar{\lambda}$, which contradicts the definition of $\bar{\lambda}$. Hence case (a) does not occur.

Next, suppose that case (c) occurs. Let $N>n_{0}$ be the point such that $\bar{y}(N-0)=\Delta \bar{y}(N-0)=\infty$. By Lemma 5, there is an $M>0$ such that solution $y(n)$ of Equation (8) satisfying $y(N) \geq 1, \Delta y(N) \geq M$ must blow up at some finite $\bar{N}=\bar{N}(y) \in(N, \infty): y(\bar{N}-0)=\Delta \bar{y}(\bar{N}-0)=\infty$. For sufficiently small $\epsilon>0$, we have $\bar{y}(N-\epsilon)>1 \quad \Delta \bar{y}(N-\epsilon)>M$. Then if $\lambda<\bar{\lambda}$ is sufficiently close to $\bar{\lambda}$, then $y_{\lambda}(n)$ can be continued at least to $N-\epsilon$, and satisfies $y_{\lambda}(N-\epsilon)>1, \Delta y_{\lambda}(N-\epsilon)>M$. Then, even through $y_{\lambda}$ can be continued to $N$, $y_{\lambda}(n)$ blows up at some finite point by the definition of $M$. This fact shows that such a $\lambda(<\bar{\lambda})$ does not belong to $S$, contradicting the definition of $\bar{\lambda}$, again. Consequently case (b) occurs, and hence the proof of Theorem 6 is complete.

Proof of Theorem 7. The proof is done by contradiction. Let $y(n)$ be a solution of Equation (1) of type (AS). We suppose that $y(n)$ exists for $n \geq n_{1}$ and satisfies

$$
y(n) \geq C_{1} n, \Delta y(n) \geq C_{1}, n \geq n_{1} \text { for some } C_{1}>0
$$

Put $z(n)=y(n)(\Delta y(n))^{\alpha}(>0) \quad n \geq n_{1}$. Then

$$
\begin{aligned}
\Delta z(n) & =y_{n+1}(\Delta y(n))^{\alpha}+y(n) \Delta(\Delta y(n))^{\alpha} \\
& =y_{n+1}(\Delta y(n))^{\alpha}+(y(n))^{\beta+1} p(n) \\
& =(\Delta y(n))^{\alpha}\left[y_{n+1}+p(n) \frac{y_{n}^{\beta+1}}{(\Delta y(n))^{\alpha}}\right] \\
& =\frac{(\Delta y(n))^{\alpha}}{y(n)}\left[\frac{y(n+1)}{y(n)}+p(n) \frac{(y(n))^{\beta}}{(\Delta y(n))^{\alpha}}\right] \\
& \geq z(n)\left[\frac{\Delta y}{y}+p(n) \frac{y^{\beta}}{(\Delta y)^{\alpha}}\right] .
\end{aligned}
$$


Now, we employ the Young inequality of the form

$$
X+Y \geq \sigma^{-\sigma}(1-\sigma)^{-(1-\sigma)} X^{1-\sigma} Y^{\sigma} \text { for } X, Y>0 \text { and } 0<\sigma<1
$$

in the last inequality. It follows therefore that

$$
\Delta z(n) \geq C_{2} z(n)(\Delta y(n))^{1-\sigma-\sigma \alpha}(y(n))^{\beta \sigma+\sigma-1}(p(n))^{\sigma}, n \geq n_{1}
$$

where $C_{2}=C_{2}(\sigma, \alpha, \beta)>0$ is a constant. We rewrite is inequality as

$$
\Delta z(n) \geq C_{2}(y(n))^{\beta \sigma-\sigma-\rho-1}(\Delta y(n))^{1-\sigma-\sigma \alpha-\rho \alpha}(p(n))^{\sigma}(z(n))^{1+\rho}, n \geq n_{1} .
$$

Noting (7.3) and condition (13), we obtain

$$
\Delta z(n) \geq C_{3} n^{\beta \sigma+\alpha-\rho-1}(p(n))^{\sigma}(z(n))^{1+\rho}, \quad n \geq n_{1},
$$

where $C_{3}=C_{3}\left(\alpha, \beta, \sigma, \rho, C_{2}, C_{1}\right)>0$ is a constant. Dividing both sides by $(z(n))^{1+\rho}$ and summing from $n$ to $\infty$, we have

$$
\frac{1}{\rho}(z(n))^{-\rho} \geq C_{3} \sum_{n}^{\infty}(s)^{\beta \sigma+\sigma-\rho-1}[p(s)]^{\sigma}, \quad n \geq n_{1}
$$

because $\lim _{n \rightarrow \infty} z(n)=\infty$. Consequently, we have

$$
\frac{1}{\rho}\left(\frac{n}{y(n)}\right)^{\rho}(\Delta y(n))^{-\alpha \rho} \geq C_{3} n^{\rho} \sum_{n}^{\infty}(s)^{\beta \alpha+\sigma-\rho-1}[p(s)]^{\sigma}, \quad n \geq n_{1} .
$$

Letting $n \rightarrow \infty$, we get a contradiction to assumption (12). This completes the proof.

As was mentioned in Section 5, the proof of Theorem 8 is omitted. In fact, a more general result is proved in Lemma 5.

\section{Proofs of Main Results for the Sub-Homogeneous Equations}

Throughout this section, we assume that $\alpha>\beta$.

Proof of Theorem 9. Let $n_{1}, n_{2}$ be fixed so that $n_{0} \leq n_{1} \leq n_{2}$ and put

$$
m=\min _{n_{1} \leq n \leq n_{2}} p(n)>0 \quad \text { and } \rho=\frac{\alpha+1}{\alpha-\beta}>0 .
$$

Then there are constants $L>0$ and $c>0$ satisfying

$$
\begin{aligned}
& L^{\beta / \alpha} \sum_{n_{1}}^{n_{2}-1}\left(\sum_{s}^{n_{2}-1} p(r)\right)^{1 / \alpha} \leq L, \\
& \frac{C^{\beta / \alpha} m^{1 / \alpha}}{(\rho \beta+1)^{1 / \alpha}} \cdot \frac{\alpha}{\alpha+\rho \beta+1} \geq c
\end{aligned}
$$

and

$$
c\left(n_{2}-n\right)^{\rho} \leq L \text { for } n_{1} \leq n \leq n_{2} .
$$

Consider the Banach space $B_{N}$ of all real sequences $y=\{y(n)\}_{n \geq N}$ with sup norm $\|y\|=\sup _{n \geq N}|y|$. Define the subset $Y$ of $B_{N}$ by

$$
Y=\left\{y^{(n)} \in C\left[n_{1}, n_{2}\right]: c\left(n_{2}-n\right)^{e} \leq y(n) \leq L \text { for } n_{1} \leq n \leq n_{2}\right\}
$$


and

$$
F y^{(n)} \begin{cases}=\sum_{n}^{n_{2}-1}\left(\sum_{s}^{n_{2}-1} p(r)[y(r)]^{\beta}\right)^{1 / \alpha}, & n_{1} \leq n \leq n_{2}, \\ =0, & \text { other wise. }\end{cases}
$$

We show that the hypothesis of the Schavder fixed point theorem is satisfied for $Y$ and $F$. Let $y \in Y$. Then, obviously $F y(n) \leq L$ for $n_{1} \leq n \leq n_{2}$. Moreover

$$
\begin{aligned}
F y(n) & \geq m^{1 / \alpha} c^{\beta / \alpha} \sum_{n}^{n_{2}-1}\left(\sum_{s}^{n_{2}-1}\left(n_{2}-r\right)^{\rho \beta}\right)^{1 / \alpha} \\
& \geq \frac{m^{1 / \alpha} c^{\beta / \alpha} \alpha}{(\rho \beta+1)^{1 / \alpha}(\alpha+\rho \beta+1)}\left(n_{2}-n\right)^{\frac{\alpha+\rho \beta+1}{\alpha}} \\
& \geq c\left(n_{2}-n\right)^{\rho}, \quad \text { for } n_{1} \leq n \leq n_{2} .
\end{aligned}
$$

Hence $F Y \subset Y$. The continuity of $F$ and the boundedness of the sets $F Y$ and $\{\Delta F Y: y \in Y\}$ can be easily established. Accordingly there is a $\bar{y} \in Y$ satisfying $F \bar{Y}=\bar{y}$. By taking difference twice, we find that $\bar{y}(n)$ is a solution of Equation (1) for $n_{1} \leq n \leq n_{2}$ and that $\bar{y}(n)>0$ for $n_{1} \leq n \leq n_{2}$ and $\bar{y}\left(n_{2}\right)=\Delta \bar{y}\left(n_{2}\right)=0$. Now, we put

$$
y(n)= \begin{cases}\bar{y}(n) & n_{1} \leq n \leq n_{2} \\ 0 & n \geq n_{2} .\end{cases}
$$

It is easy to see that $y(n)$ is a solution of equation (8) for $n \geq n_{1}$ and is of type $\left(S_{1}\right)$. The proof is complete.

Theorems 14 and 15 can be proved easily as in the proofs of Theorems 4 and 5 respectively. We therefore omit the proofs.

Proof of Theorem 10. By our assumption we can find a positive solution $y_{k}(n), k \in N$ of Equation (8) satisfying $y_{k}(\infty)=\frac{1}{K}$. Since $\alpha>\beta$, we see by Lemma 4 that each $y_{k}(n)$ exists for $n \geq n_{0}$. We show that the sequence $\left\{y_{k}(n)\right\}$ has the limit function $y(n)$, and it gives rise to a positive solution of Equation (8) of type (D).

We first claim that

$$
y_{1}(n)>y_{2}(n)>\cdots>y_{k}(n)>y_{k+1}(n)>\cdots>0, n \geq n_{0} .
$$

If this is not true, then $y_{i}(\bar{n})=y_{i+1}(\bar{n})$ for some $i \in N$ and $\bar{n} \geq n_{0}$. This means however that there are two nonnegative nonincreasing solutions of Equation (8) passing through the point $\left(\bar{n}, y_{i}(\bar{n})\right)$. This contradiction to Theorem 17. We therefore have (38) and so $\lim _{k \rightarrow \infty} y_{k}(n)=y(n)$ exists observe that $y_{k}(n)$ satisfies

$$
y_{k}(n)=\frac{1}{K}+\sum_{n}^{\infty}\left(\sum_{s}^{\infty} p(r)\left[y_{i}(r)\right]^{\beta}\right)^{1 / \alpha}, \quad n \geq n_{0} .
$$

Letting $k \rightarrow \infty$, we obtain via the dominated convergence theorem

$$
y(n)=\sum_{n}^{\infty}\left(\sum_{s}^{\infty} p(r)[y(r)]^{\beta}\right)^{1 / \alpha}, \quad n \geq n_{0} .
$$

We see that $y(n)$ is a nonnegative solution of Equation (8) satisfying $y(\infty)=0$. It remains to prove that $y(n)>0$ for $n \geq n_{0}$ Fix $N>n_{0}$ arbitrarily. The proof of Theorem 2 implies that there is a solution $y_{N}(n)>0$ for $n_{0} \leq n<N$ and $y_{N}(n)=0$ for $n \geq N$. We claim that

$$
y_{k}(n)>y_{N}(n) \text { for } n_{0} \leq n \leq N \text { for all } k \in N .
$$

In fact, if this fails to hold, then 


$$
y_{i}(\bar{n})=y_{N}(\bar{n}) \text { for some } i \in N \text { and } n_{0} \leq \bar{n}<N .
$$

By this means, as before, that there are two nonnegative nonincreasing solution fo Equation (8) passing through the point $\left(\bar{n}, y_{N}(\bar{n})\right)$. This contradiction shows that (39) holds. Hence by letting $i \rightarrow \infty$ in (39) we have $y(n) \geq y_{N}(n)>0$ for $n_{0} \leq n<N$. Since $N>n_{0}$ is arbitrary, we see that $y(n)>0$ for $n \geq n_{0}$. The proof is complete.

Proof of Theorem 11. The proof is done by contradiction. Let $y(n)$ be a positive solution of equation (8) for $n \geq n_{0}$ of type (D). Using (16). We obtain from Equation (8)

$$
\Delta(\Delta y(n))^{\alpha} \geq C_{1} n^{-\alpha}(y(n))^{\beta}, \quad n \geq n_{1} .
$$

where $C_{1}$ is a positive constant. We fix a $N \geq n_{1}$ arbitrary and consider inequality (42) only on the interval $[N, 2 N]$ for a moment. A summation of (42) from $n$ to $2 N$, given

$$
\begin{aligned}
& \left(\Delta y(2 N+1)^{\alpha}\right)-(\Delta y(n))^{\alpha} \geq C_{1} \sum_{n}^{2 N} s^{-\alpha}(y(s))^{\beta} \\
& -(\Delta y(n))^{\alpha} \geq C_{1} \frac{1}{N^{\alpha}}(y(2 N))^{\beta}-(y(n))^{\beta} \\
& -\Delta y(n) \geq C_{1} \frac{1}{N}\left[(y(2 N))^{\beta}-(y(n))^{\beta}\right]^{\frac{1}{\alpha}}, \quad N \leq n \leq 2 N \\
& \frac{-\Delta y(n)}{\left[(y(2 N))^{\beta}-(y(n))^{\beta}\right]^{\frac{1}{\alpha}}} \geq C_{1} N^{-1}, \quad N \leq n \leq 2 N .
\end{aligned}
$$

From which, we have

$$
\begin{aligned}
& \int_{y(N)}^{y(2 N)} \frac{\mathrm{d} u}{\left[u^{\beta}-(y(2 N))^{\beta}\right]^{\frac{1}{\alpha}}} \geq C_{2}, \quad N \geq n_{1} \\
& (y(2 N))^{\frac{\alpha-\beta}{\alpha}} \int_{y(N) / y(2 N)}^{1}\left(v^{\beta}-1\right)^{\frac{\alpha-\beta}{\alpha}} \mathrm{d} v \geq C_{2}, \quad N \geq n_{1} .
\end{aligned}
$$

We can find a constant $C>0$ satisfying

$$
\int_{x}^{1}\left(v^{\beta}-1\right)^{-\frac{1}{\alpha}} \mathrm{d} v \leq C(1-x)^{\frac{\alpha-\beta}{\alpha+1}}, \quad x \rightarrow 0 .
$$

Therefore (43) implies that

$$
C(y(2 N))^{\frac{\alpha-\beta}{\alpha}}\left[1-\left(\frac{y(N)}{y(2 N)}\right)\right]^{\frac{\alpha-\beta}{\alpha}} \geq C_{2}, \quad N \geq n_{1}
$$

from which we have

$$
C[(y(2 N))-(y(N))]^{\frac{\alpha-\beta}{\alpha}} \geq C_{2}, \quad N \geq n_{1} .
$$

Letting $N \rightarrow \infty$, we have a contradiction. The proof is complete.

Proof of Theorem 12. The proof is done by contradiction. Let $y(n)$ be a solution of Equation (8) of type (D). We notice first that

$$
\lim _{n \rightarrow \infty} n \Delta y(n)=0 .
$$

In fact, since $\Delta^{2} y(n)>0$, we can compute as follows 


$$
\begin{aligned}
\Delta y(n) & =\sum_{n}^{\infty}(-\Delta y(s)) \geq \sum_{n}^{2 n}(-\Delta y(s)) \\
& \geq-\Delta y(2 n) \sum_{n}^{2 n} p(s)=-\Delta y(2 n) \cdot n \geq 0 \quad \text { for large } n .
\end{aligned}
$$

Therefore (42) holds.

We may suppose that for some $C_{1}>0$ and $n_{1} \geq n_{0}$

$$
0<y(n) \leq C_{1}, \quad 0<-n \Delta y(n) \leq C_{1}, \quad n \geq n_{1} .
$$

But $z(n)=y(-\Delta y)^{\alpha}(>0) n \geq n_{1}$. Then

$$
\begin{aligned}
-\Delta z(n) & =(-\Delta y(n))(-\Delta y(n))^{\alpha}-y_{n+1} \Delta\left(-\Delta y_{n}\right)^{\alpha} \\
& =(-\Delta y(n))^{\alpha+1}+y_{n+1} p(n)(y(n))^{\beta} \\
& =y(-\Delta y(n))^{\alpha}\left[\frac{(-\Delta y(n))}{y(n)}+p(n)(y(n))^{\beta}\left(\frac{y(n)}{-\Delta y(n)}-1\right)\right] \\
& \geq y(-\Delta y(n))^{\alpha}\left[\frac{(-\Delta y(n))}{y(n)}+p(n) \frac{(y(n))^{\beta}}{(-\Delta y(n))^{\alpha}}\right]
\end{aligned}
$$

proceeding as in the proof of Theorem 8 , we obtain

$$
-\Delta z(n) \geq C_{2}(y(n))^{\sigma+\sigma \alpha-\rho \alpha-1}(-\Delta y(n))^{1-\sigma-\sigma \alpha+\rho \sigma}[p(n)]^{\alpha}(z(n))^{1-\rho}, \quad n \geq n_{1}
$$

where $C_{2}$ is a constant. We obtain from (43) and assumption (18)

$$
-\Delta z(n) \geq C_{3} n^{\sigma+\sigma \alpha-\rho \alpha-1}[p(n)]^{\sigma}(z(n))^{1-\rho}, \quad n \geq n_{1}
$$

where $C_{3}>0$ is a constant. Dividing both sides by $(z(n))^{1-\rho}$ and summing from $n$ to $\infty$, we have

$$
\frac{1}{\rho}(z(n))^{\rho} \geq C_{3} \sum_{n}^{\infty}(s)^{\sigma+\sigma \alpha-\rho \alpha-1}[p(s)]^{\sigma}, \quad n \geq n_{1}
$$

that is,

$$
\frac{1}{\rho}(y(n))^{\rho}(-n \Delta y(n))^{\sigma \rho} \geq C_{3} n^{\sigma \rho} \sum_{n}^{\infty}(s)^{\sigma+\sigma \alpha-\rho \alpha-1}[p(s)]^{\sigma}, \quad n \geq n_{1} .
$$

Letting $n \rightarrow \infty$, we get a contradiction to assumption (17) by (42). The proof is complete.

Proof of Theorem 16. Sufficiency Part: By Theorem 17 and (2) of Remark 6.2, there is a positive solution $y(n)$ of equation (8) satisfying $y(\infty)=\infty$. This $y(n)$ is either of type $(A L)$ or of type (AS). But by Theorem 15, we see that $y(n)$ must be of type $(A S)$.

Necessity Part: Let $y(n)$ be a positive solution of Equation (8) for $n \geq n_{1}$ of type (AS). To prove (19), we suppose the contrary that $\sum^{\infty} n^{\beta} p(n)<\infty$. As in the proof of Lemma 5.3, we have

$$
|y(n)|=\left|\rho_{0}\right|+\sum_{n_{1}}^{n-1}\left(\left|C_{1}\right|^{\alpha}+\sum_{n_{1}}^{s-1} p(r)|y(r)|^{\beta}\right)^{1 / \alpha}, n \geq n_{1}
$$

where $c_{0}=y\left(n_{1}\right)$ and $c_{1}=\Delta y\left(n_{1}\right)$ let $z(n)=\max _{n_{1} \leq \xi \leq n-1} \frac{|y(\xi)|}{\xi}$. It follows that 


$$
\begin{aligned}
\frac{|y(n)|}{n} & \leq C_{2}+\frac{1}{n} \sum_{n_{1}}^{n-1}\left(\left|c_{1}\right|^{\alpha}+[z(s)]^{\beta} \sum_{n_{1}}^{s-1} r^{\beta} p(r)\right)^{1 / \alpha} \\
& \leq c_{2}+\left(\left|c_{1}\right|^{\alpha}+[z(s)]^{\beta} \sum_{n_{1}}^{n-1} r^{\beta} p(r)\right)^{1 / \alpha}, n \geq n_{1}
\end{aligned}
$$

where $c_{2}$ is a constant. put $w(n)=\max \left\{\left|c_{1}\right|^{\alpha / \beta}, z(n)\right\}$. We then have

$$
z(n) \leq c_{2}+[w(n)]^{\beta / \alpha}\left(1+\sum_{n_{1}}^{n-1} r^{\beta} f(r)\right)^{1 / \alpha}, \quad n \geq m .
$$

Since $y(n)$ is of type (AS), $\frac{|y(n)|}{n}$ is unbounded for $n \geq n_{1}$ and so is $z(n)$. Accordingly, there is a $n_{2} \geq n$, satisfying $w(n) \equiv z(n)$ for $n \geq n_{2}$. Thus

$$
\begin{aligned}
w(n) & \leq c_{2}+(w(n))^{\beta / \alpha}\left(1+\sum_{n_{1}}^{n-1} r^{\beta} p(r)\right)^{1 / \alpha} \\
& \leq c_{2}+(w(n))^{\beta / \alpha}\left(1+\sum_{n_{1}}^{\infty} r^{\beta} p(r)\right)^{1 / \alpha}, n \geq n_{2} .
\end{aligned}
$$

Since $\beta / \alpha<1$, this implies the boundedness of $w$, which is a contraction. Hence, we must have (19). The proof is complete.

Theorem 16 is clear because of all solutions of equation (8) with $\alpha<\beta$ exist for $n \geq n_{0} \quad$ [see Lemma 5].

\section{References}

[1] Mizukami, M., Naito, M. and Usami, H. (2002) Asymtotic Behavior of Solutions of a Class of Second Order Quasilinear Ordinary Differential Equations. Hiroshima Mathematical Journal, 32, 51-78.

[2] Tiryaki, A. (2012) Some Criteria for the Asymptotic Behavior of a Certain Second Order Nonlinear Perturbed Differential Equations. Advances in Pure Mathematics, 2, 341-343. http://dx.doi.org/10.4236/apm.2012.25048

[3] Drozdowica, A. and Popenda, J. (1987) Asymptotic Behavior of the Solutions of the Second Order Difference Equation. Proceedings of the American Mathematical Society, 99, 135-140. http://dx.doi.org/10.1090/S0002-9939-1987-0866443-0

[4] Drozdowica, A. and Popenda, J. (1993) Asymptotic Behavior of Solutions of Difference Equations of Second Order. Journal of Computational and Applied Mathematics, 47, 141-149. http://dx.doi.org/10.1016/0377-0427(93)90001-R

[5] Elbert, A. and Kwsano, T. (1990) Oscillation and Nonoscillation Theorems for a Class of Second Order Quailinear Differential Equations. Acta Mathematica Hungarica, 56, 325-336. http://dx.doi.org/10.1007/BF01903849

[6] He, X.Z. (1993) Oscillatory and Asymptotic Behavior of Second Order Nonlinear Difference Equations. Journal of Mathematical Analysis and Applications, 175, 482-498. http://dx.doi.org/10.1006/jmaa.1993.1186

[7] Kiguradze, I.T. and Chanturia, T.A. (1990) Asymptotic Properties of Solutions of Non Autonomous Ordinary Differential Equations. Nauka, Moscow.

[8] Kubiaczyk, I. and Sekar, S.H. (2002) Oscillation Theorems for Second Order Sublinear Delay Difference Equations. Mathematica Slovaca, 52, 343-359.

[9] Kusano, T., Naito, K. and Ogata, A. (1994) Strong Oscillations and Nonoscillation of Quasilinear Differential Equations of Second Order. Differential Equations and Dynamical Systems, 2, 1-10.

[10] Luo, J. (2002) Oscillation Criteria for Second Order Quasilinear Neutral Difference Equations. Computers \& Mathematics with Applications, 43, 1549-1557. http://dx.doi.org/10.1016/S0898-1221(02)00118-9

[11] Migda, M. and Migda, J. (1998) Asymptotic Behavior of the Solutions of the Second Order Difference Equations. Archivum Mathematicum, 34, 467-476.

[12] Sadhasivam, V., Sundar, P. and Santhi, A. (2016) Oscillation and Asymptotic Behavior of Solutions of Second Order Homogeneous Neutral Difference Equations with Positive and Negative Coefficients. IOSR Journal of Mathematics, 12, 36-42. 
[13] Thandapani, E. (1992) Asymptotic and Oscillatory Behavior of Solutions of Second Order Nonlinear Neutral Delay Difference Equations. Rivista di Matematica della Università di Parma, 1, 105-133.

[14] Thandapani, E. and Arul, R. (1997) Oscillation Theory for a Class of Second Order Quasilinear Difference Equation. Tamkang Journal of Mathematics, 28, 229-238.

[15] Thandapani, E., Graef, J.R. and Spikes, P.W. (1996) On the Oscillation of Solutions of Second Order Quasilinear Difference Equations. Nonlinear World, 3, 545-565.

[16] Wong, P.J.Y. and Agarwal, R.P. (1996) Oscillation Theorems for Certain Second Order Nonlinear Difference Equations. Journal of Mathematical Analysis and Applications, 204, 813-829. http://dx.doi.org/10.1006/jmaa.1996.0469

[17] Wong, P.J.W. and Agarwal, R.P. (1996) Oscillation and Monotone Solutions of a Second Order Quasilinear Difference Equation. Funkcialaj Ekvacioj, 39, 491-517.

[18] Yan, J. and Liu, B. (1995) Asymptotic Behavior of Nonlinear Delay Difference Equations. Applied Mathematics Letters, 8, 1-5. http://dx.doi.org/10.1016/0893-9659(95)00075-2

[19] Rath, R.N., Seshadev, P. and Barik, B.L.S. (2008) Oscillatory and Asymptotic Behaviour of a Homogeneous Neutral Delay Difference Equations of Second Order. Bulletin of the Institute of Mathematics Academia Sinica (New Series), 3, 453-466.

[20] Agarwal, R.P. (1992) Difference Equations and Inequalities Theory, Method and Applications. Marcel Dekker, New York.

[21] Elaydi, S.N. (1996) An Introduction to Difference Equations. Springer Verlag, New York. http://dx.doi.org/10.1007/978-1-4757-9168-6

[22] Gyori, I. and Ladas, G. (1991) Oscillation Theory of Delay Differential Equations with Applications. Clarendon Press, Oxford.

[23] Kellay, W.G. and Peterson, A.C. (1991) Difference Equations: An Introduction with Applications. Academic Press, New York.

[24] Lakshmikanthan, V. and Trigiante, O. (1988) Theory of Difference Equations: Numerical Method and Application. Academic Press, New York.

[25] Wong, P.J.Y. and Agarwal, R.P. (1996) On the Oscillation and Asymptotically Monotone Solutions of Second Order Quasilinear Difference Equations. Applied Mathematics and Computation, 79, 207-237. http://dx.doi.org/10.1016/0096-3003(95)00267-7

[26] Thandapani, E., Manuel, M.M.S. and Agarwal, R.P. (1996) Oscillation and Non Oscillation Theorems for Second Order Quasilinear Difference Equations. Facta Universitatis, Series: Mathematics and Informatics, 11, 49-65.

[27] Sundar, P. and Thandapani, E. (2000) Oscillation and Non-Oscillation Theorems for Second Order Quasilinear Functional Difference Equations. Indian Journal of Pure and Applied Mathematics, 31, 37-47.

[28] Thandapani, E. and Arul, R. (1997) Oscillation and Nonoscillation Theorems for a Class of Second Order Quasilinear Difference Equations. Zeitschrift Für Analysis Und Ihre Anwendungen, 16, 749-759. http://dx.doi.org/10.4171/ZAA/789

[29] Thandapani, E., Liu, Z., Arul, R. and Raja, P.S. (2004) Oscillation and Asymptotic Behavior of Second Order Difference Equations with Nonlinear Neutral Terms. Applied Mathematics E-Notes, 4, 59-67.

[30] Cheng, S.S. and Patula, W.T. (1993) An Existence Theorem for a Nonlinear Difference Equations. Nonlinear Analysis, Theory Method and Applications, 20, 193-203. http://dx.doi.org/10.1016/0362-546X(93)90157-N 


\section{Submit or recommend next manuscript to SCIRP and we will provide best service for you:}

Accepting pre-submission inquiries through Email, Facebook, LinkedIn, Twitter, etc.

A wide selection of journals (inclusive of 9 subjects, more than 200 journals)

Providing 24-hour high-quality service

User-friendly online submission system

Fair and swift peer-review system

Efficient typesetting and proofreading procedure

Display of the result of downloads and visits, as well as the number of cited articles

Maximum dissemination of your research work

Submit your manuscript at: http://papersubmission.scirp.org/ 